Louisiana State University

LSU Digital Commons

7-14-2005

\title{
Probing temporal aspects of high-order harmonic pulses via multi- colour, multi-photon ionization processes
}

\author{
J. Mauritsson \\ Louisiana State University \\ P. Johnsson \\ Lunds Tekniska Högskola \\ R. López-Martens \\ Lunds Tekniska Högskola \\ K. Varjú \\ Lunds Tekniska Högskola \\ A. L'Huillier \\ Lunds Tekniska Högskola
}

See next page for additional authors

Follow this and additional works at: https://digitalcommons.Isu.edu/physics_astronomy_pubs

\section{Recommended Citation}

Mauritsson, J., Johnsson, P., López-Martens, R., Varjú, K., L'Huillier, A., Gaarde, M., \& Schafer, K. (2005).

Probing temporal aspects of high-order harmonic pulses via multi-colour, multi-photon ionization processes. Journal of Physics B: Atomic, Molecular and Optical Physics, 38 (13), 2265-2278.

https://doi.org/10.1088/0953-4075/38/13/018

This Article is brought to you for free and open access by the Department of Physics \& Astronomy at LSU Digital Commons. It has been accepted for inclusion in Faculty Publications by an authorized administrator of LSU Digital Commons. For more information, please contact ir@lsu.edu. 


\section{Authors}

J. Mauritsson, P. Johnsson, R. López-Martens, K. Varjú, A. L'Huillier, M. B. Gaarde, and K. J. Schafer 


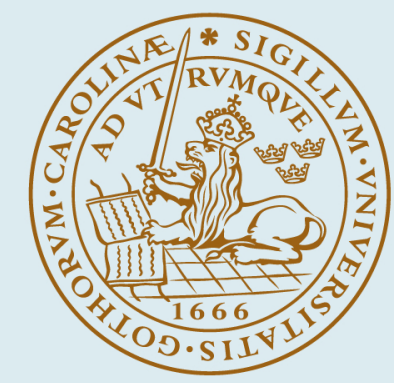

\section{LUND UNIVERSITY}

\section{Probing temporal aspects of high-order harmonic pulses via multi-colour, multi-photon ionization processes}

Mauritsson, J; Johnsson, Per; Lopez, Rodrigo; Varju, Katalin; L'Huillier, Anne; Gaarde, MB; Schafer, KJ

Published in:

Journal of Physics B: Atomic, Molecular and Optical Physics

DOI:

10.1088/0953-4075/38/13/018

2005

Link to publication

Citation for published version (APA):

Mauritsson, J., Johnsson, P., Lopez, R., Varju, K., L'Huillier, A., Gaarde, MB., \& Schafer, KJ. (2005). Probing temporal aspects of high-order harmonic pulses via multi-colour, multi-photon ionization processes. Journal of Physics B: Atomic, Molecular and Optical Physics, 38(13), 2265-2278. https://doi.org/10.1088/09534075/38/13/018

Total number of authors:

7

\footnotetext{
General rights

Unless other specific re-use rights are stated the following general rights apply:

Copyright and moral rights for the publications made accessible in the public portal are retained by the authors and/or other copyright owners and it is a condition of accessing publications that users recognise and abide by the legal requirements associated with these rights.

- Users may download and print one copy of any publication from the public portal for the purpose of private study or research.

- You may not further distribute the material or use it for any profit-making activity or commercial gain

- You may freely distribute the URL identifying the publication in the public portal

Read more about Creative commons licenses: https://creativecommons.org/licenses/

Take down policy

If you believe that this document breaches copyright please contact us providing details, and we will remove access to the work immediately and investigate your claim.
} 
Probing temporal aspects of high-order harmonic pulses via multi-colour, multi-photon ionization processes

This article has been downloaded from IOPscience. Please scroll down to see the full text article.

2005 J. Phys. B: At. Mol. Opt. Phys. 382265

(http://iopscience.iop.org/0953-4075/38/13/018)

View the table of contents for this issue, or go to the journal homepage for more

Download details:

IP Address: 130.235.188.104

The article was downloaded on 05/07/2011 at 07:00

Please note that terms and conditions apply. 


\title{
Probing temporal aspects of high-order harmonic pulses via multi-colour, multi-photon ionization processes
}

\author{
J Mauritsson ${ }^{1}$, P Johnsson ${ }^{2}$, R López-Martens ${ }^{2}$, K Varjú ${ }^{2}$, A L'Huillier ${ }^{2}$, \\ M B Gaarde ${ }^{1}$ and K J Schafer ${ }^{1}$ \\ ${ }^{1}$ Department of Physics and Astronomy, Louisiana State University, Baton Rouge, \\ LA 70803-4001, USA \\ ${ }^{2}$ Department of Physics, Lund Institute of Technology, PO Box 118, SE-22100 Lund, Sweden \\ E-mail: mauritsson@phys.lsu.edu
}

Received 21 March 2005, in final form 22 March 2005

Published 14 June 2005

Online at stacks.iop.org/JPhysB/38/2265

\begin{abstract}
High-order harmonics generated through the interaction of atoms and strong laser fields are a versatile, laboratory-scale source of extreme ultraviolet (XUV) radiation on a femtosecond or even attosecond time-scale. In order to be a useful experimental tool, however, this radiation has to be well characterized, both temporally and spectrally. In this paper we discuss how multi-photon, multi-colour ionization processes can be used to completely characterize either individual harmonics or attosecond pulse trains. In particular, we discuss the influence of the intensity and duration of the probe laser, and how these parameters effect the accuracy of the XUV characterization.
\end{abstract}

\section{Introduction}

The production of extreme ultraviolet (XUV) radiation through high-order harmonic generation [1] is currently a very active field of research [2]. This is because the harmonic pulses offer unprecedented time resolution in the XUV wavelength regime, which makes them complementary to longer pulsed synchrotron sources. The generated harmonic spectrum consists of a comb of frequencies, separated by twice the fundamental frequency, up to a characteristic cutoff energy [3]. On the one hand, each tooth of the comb corresponds in time to a pulse of femtosecond (fs, $10^{-15} \mathrm{~s}$ ) duration [4-6]; while on the other hand, the total comb may correspond to a train of bursts with attosecond (as, $10^{-18} \mathrm{~s}$ ) duration [7-12]. Isolated, single attosecond pulses can be produced if the harmonic emission is confined to less than half an optical cycle of the driving field [13]. Both individual harmonics and attosecond pulses enable many new time-resolved experiments [14-16] and are also a useful tool to probe electron continuum dynamics [17]. 
In this paper we discuss different methods to measure the duration and the time-frequency modulation of the harmonic emission based on photoelectron spectroscopy. The total harmonic field, $\tilde{\varepsilon}_{\mathrm{XUV}}(t)$, is a coherent sum of the individual harmonics:

$$
\tilde{\varepsilon}_{\mathrm{XUV}}(t)=\sum_{q} \tilde{\varepsilon}_{q}(t)=\sum_{q} F_{q}(t) \mathrm{e}^{-\mathrm{i}\left[q \omega_{1} t+\Phi_{q}(t)\right]},
$$

where $F_{q}(t)$ is the electric field envelope of the $q$ th harmonic, $\Phi_{q}(t)$ is the harmonic phase and $\omega_{1}$ is the fundamental driving frequency. When considering the time-frequency structure of the harmonic emission, it is useful to separate the total harmonic phase $\Phi_{q}(t)$ into a constant term and a time-varying term:

$$
\Phi_{q}(t)=\phi_{q}^{0}+\phi_{q}(t),
$$

where the absolute phase $\phi_{q}^{0}$ is chosen so that the temporal phase $\phi_{q}(t)$ is zero for $t=0$. For individual harmonics, $\phi_{q}^{0}$ is only an absolute phase shift of the carrier wave, and their time-frequency structure is determined solely by $F_{q}(t)$ and $\phi_{q}(t)$. For an attosecond pulse train (APT), however, the shape and duration of the bursts are determined by the variation of $F_{q}(t)$ and $\Phi_{q}(t)$ with $q$, since the q dependence determines the phase-locking of the different harmonics. Therefore, the absolute phase $\phi_{q}^{0}$ determines the average shape of the bursts, while the temporal phase, $\phi_{q}(t)$, may alter the shape from pulse to pulse in the APT $[6,18]$. In order to completely characterize the harmonic emission $F_{q}(t), \phi_{q}^{0}$ and $\phi_{q}(t)$ have to be measured simultaneously.

For high-order harmonic emission, the time-dependent frequency consists mainly of a linear chirp [19]. This applies to both single harmonics [6] and attosecond pulses [8, 11] synthesized from several harmonics. For the $q$ th harmonic, the time-dependent frequency is given by

$$
\omega_{q}(t)=q \omega_{1}+\frac{\mathrm{d} \phi_{q}(t)}{\mathrm{d} t}=q \omega_{1}+b_{q} t+O\left(t^{2}\right),
$$

where the chirp rate $b_{q}$ is used to describe the linear frequency modulation. For the attosecond bursts, the important parameter is the variation of $\phi_{q}^{0}$ with $q$. Defining the absolute phase difference between consecutive harmonics as $\Delta \phi_{q+1}^{0}=\phi_{q}^{0}-\phi_{q+2}^{0}$, this is proportional to the average group delay of the set of harmonics centred around the frequency $(q+1) \omega_{1}$, and a variation of $\Delta \phi_{q+1}^{0}$ with $q$ leads to a time-dependent frequency modulation on each attosecond burst.

The frequency modulation of the harmonic emission is imposed by the non-trivial electron dynamics in the generation process [20,21]. While this has long been understood theoretically [22], the tools needed to experimentally measure ultrashort pulses in the XUV wavelength region have only recently been developed [4-7, 23, 24]. The experimental measurement techniques are almost all based on photoelectron spectroscopy, most often using crosscorrelations between the unknown XUV pulse(s) and a known IR pulse. The cross-correlations are performed in a pump-probe arrangement, and we will refer to the XUV pulses as the pump and the dressing IR field as the probe from here on. The needed synchronization between the pump and the probe is usually achieved by using the same IR field to both generate the harmonics and perform the cross-correlation.

In the perturbative regime, the presence of a moderately strong IR field modifies the XUV photoelectron spectra in two ways. First, it adds sidebands to the main XUV absorption peaks [25-27]. These sidebands are the result of laser induced transitions from one continuum state to another by the additional absorption or emission of an IR photon. Second, it induces a ponderomotive shift (AC Stark shift), which increases the ionization potential, thereby lowering the photoelectron energy of the absorption peaks. While the first effect is desirable, 
the second may introduce difficulties when extracting the XUV pulse information from the sideband signal. Both effects depend on the IR intensity. Increasing the intensity increases the visibility of the sidebands, making the cross-correlation measurement easier. However, it also yields a larger ponderomotive shift, which complicates the data analysis.

In the first section of this paper, we study the case where only one harmonic contributes to the ionization in the presence of a moderately strong IR field. We present a simple model incorporating the two intensity effects discussed above and show how the time-frequency structure (i.e. $F_{q}(t)$ and $\phi_{q}(t)$ ) of the XUV pulse can be retrieved from the energy-resolved sideband signal. We also study the ponderomotive shift with special emphasis on the important ratio between the durations of the XUV and IR pulses. If the same pulse that generates the harmonics is used as a probe, the probe will inevitably be longer than the XUV pulse, due to the non-linearity of the harmonic generation process. It is, however, possible to have an IR pulse with a duration shorter than that of the XUV if the two pulses are manipulated independently [6]. We show that the effect of the ponderomotive shift of the sidebands is significantly reduced using a shorter IR probe, thereby increasing the accuracy of the measurement.

In the second section of this paper, we expand the model to include several harmonics which can form an APT if properly synchronized. Since the harmonics are separated by $2 \omega_{1}$, where $\omega_{1}$ is both the frequency of the fundamental pulse driving the generation and also the frequency of the probe, each sideband will now receive contributions from two consecutive harmonics. The different contributions to the same sideband interfere, and the sideband signal will be modulated as a function of the time-delay, $\Delta t$, between the pump and the probe. From this modulation the absolute phase difference, $\Delta \phi_{q+1}^{0}=\phi_{q}^{0}-\phi_{q+2}^{0}$, between successive harmonics at $(q+2) \omega_{1}$ and $q \omega_{1}$ can be retrieved [7]. Knowing $\Delta \phi_{q+1}^{0}$ and the easily measured harmonic intensities, the time structure of the harmonic emission can be reconstructed. So far, reconstructions based on this procedure have assumed that each harmonic consists of only one frequency (i.e. $\phi_{q}(t)=0$ ). In this case the reconstructed time structure will, therefore, only be the average time structure of the pulses in the APT [24]. In order to accurately characterize the total harmonic radiation, both $\phi_{q}(t)$ and $\phi_{q}^{0}$ have to be measured simultaneously, together with $F_{q}(t)$. We show that one way of doing this is to combine the two methods and analyse the time-gated energy-resolved sidebands (TIGERS).

\section{Two-colour, multi-photon photoelectron spectra}

In this section we analyse the photoelectron spectra resulting from the ionization of an atom by only one harmonic (with sufficient energy for one photon ionization) in the presence of a moderately strong IR pulse. We start without the IR field, in which case the photoelectron spectrum can easily be calculated from first-order time-dependent perturbation theory. Using the dipole approximation, the amplitude of the photoelectron peak, $\mathcal{A}(\omega)$, can be expressed as the Fourier transform of the time-dependent XUV field, $\tilde{\varepsilon}_{q}(t)$, shifted by the ionization potential, $I_{p}$, of the target atom. We use atomic units, unless otherwise stated. The amplitude is

$$
\mathcal{A}(\omega)=\int_{-\infty}^{\infty} \mathrm{d} t \mathrm{e}^{\mathrm{i} \omega t} \tilde{\mu}_{\mathrm{qg}} \tilde{\varepsilon}_{q}(t) \mathrm{e}^{\mathrm{i} I_{p} t}
$$

where $\tilde{\mu}_{\mathrm{qg}}=\left|\tilde{\mu}_{\mathrm{gg}}\right| \mathrm{e}^{-\mathrm{i} \phi_{q}^{\mathrm{at}}}$ is the complex ground state-to-continuum dipole matrix element. The characteristic atomic phase $\phi_{q}^{\text {at }}$ [28] does not affect the one photon absorption spectrum since $I(\omega)=|\mathcal{A}(\omega)|^{2}$, but it becomes important for the multi-colour, multi-photon ionization, described in section 3 , where different quantum paths lead to the same final state. 


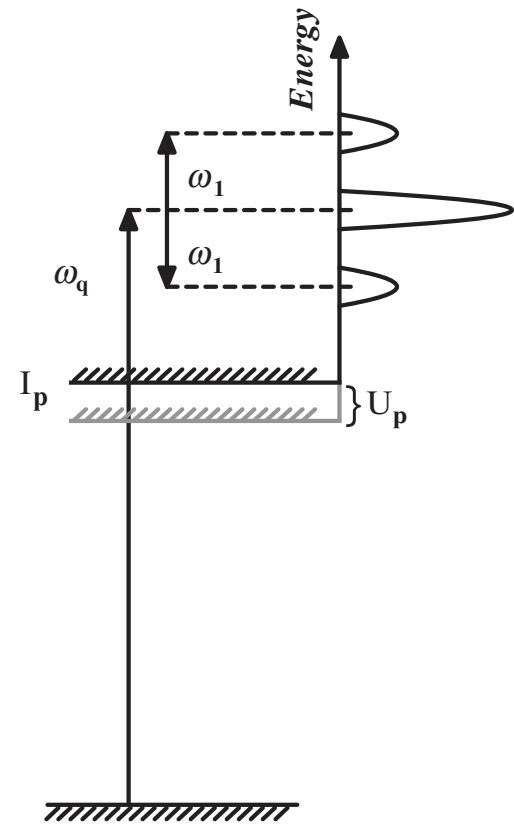

Figure 1. Schematic of two-colour, two-photon ionization. The presence of the IR field during the ionization modifies the XUV photoelectron spectrum in two ways. Sidebands are added to the main peak and the ponderomotive shift of the ionization potential decreases the final electron energy.

The presence of an IR pulse during the ionization process alters the photoelectron spectrum in, mainly, two ways: (1) the photoelectron peaks are shifted in energy due to the ponderomotive increase of the ionization potential and (2) sidebands appear on both sides of the principal peak due to the additional absorption or emission of an IR photon, as illustrated in figure 1.

Let us first examine the effect of the IR pulse on the main absorption peak, before introducing the sidebands. The presence of an IR field during the ionization process leads to an increase of the effective ionization potential of the atoms [29, 30]. This effect can be understood as upon ionization the electron must acquire sufficient energy to overcome the ionization potential, but also have sufficient energy to quiver in the field. The quiver energy is not supplied by the IR field but instead by the XUV photon resulting in a lower average electron kinetic energy [26]. This can be included in the simple model (equation (4)) by adding a time-dependent phase $\Phi_{P}(t)$ :

$$
\mathcal{A}(\omega, \Delta t)=\int_{-\infty}^{\infty} \mathrm{d} t \mathrm{e}^{\mathrm{i} \omega t} \tilde{\mu}_{\mathrm{qg}} \tilde{\varepsilon}_{q}(t) \mathrm{e}^{\mathrm{i} I_{p} t} \mathrm{e}^{\mathrm{i} \Phi_{P}(t-\Delta t)},
$$

where $\Delta t$ is the time-delay between the IR and the XUV pulses. The phase, $\Phi_{P}(t)$, is given by $\Phi_{P}(t)=\alpha \int_{-\infty}^{t} \mathrm{~d} t^{\prime} I_{\mathrm{IR}}\left(t^{\prime}\right)$, where the ponderomotive coefficient $\alpha$ is $93.3 \lambda^{2} \mathrm{meV}^{\mathrm{TW}}{ }^{-1}$ $\mathrm{cm}^{-2}$ if the wavelength is in $\mu \mathrm{m}$. The IR probe does not necessarily have the same duration as the XUV pulse, and the relative duration between the pulses turns out to be important when describing the ponderomotive effect. In most experiments the IR probe is longer than the XUV pulse in which case $\Phi_{P}(t)$ will vary almost linearly across the duration of the XUV pulse. A linear phase variation results only in an energy shift of the harmonic peak. At $\Delta t=0$ this shift is maximized. The variation of the harmonic peak as a function of time-delay is depicted 


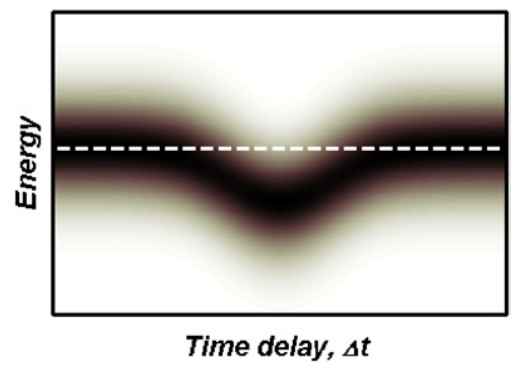

Figure 2. Ponderomotive shift of the main ionization peak as a function of time-delay, $\Delta t$, between the pump and the probe. The white, dashed line corresponds to the centre of the peak when no IR pulse is present.

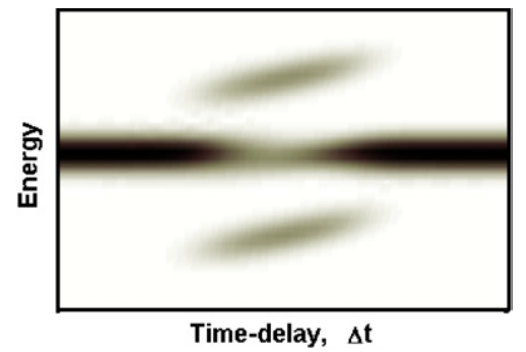

Figure 3. Sidebands as a function of time-delay, $\Delta t$, between the pump and the probe, where the pump has a linear chirp. The chirp on the XUV pump results in a tilt of the sideband, i.e. a linear variation in energy as a function of time-delay.

for this pulse configuration in figure 2. If the IR pulse is shorter than the XUV pulse, most of the ionization occurs without the presence of the IR. In this case $\Phi_{P}(t)$ is best described as a phase modulation, thereby changing the structure of the resulting peak but not significantly its position. There are many interesting effects with regard to this reshaping of the spectrum, but it is outside the scope of this paper and will be published elsewhere [37].

Even though the ponderomotive shift can be used to estimate the duration of the XUV pulse [33], the emphasis in this paper is on how to use the sidebands to extract the time-frequency structure of the XUV pulse(s). We therefore need a simple model which includes all the sideband features that are essential for the time-frequency characterization. The amplitude of the first-order sidebands can be calculated if the real IR field, $\varepsilon_{\mathrm{IR}}(t)=F_{\mathrm{IR}}(t)\left(\mathrm{e}^{\mathrm{i} \omega_{1} t}+\mathrm{e}^{-\mathrm{i} \omega_{1} t}\right)$, is added to the model

$$
\mathcal{A}_{\mathrm{SB}}(\omega, \Delta t)=\int_{-\infty}^{\infty} \mathrm{d} t \mathrm{e}^{\mathrm{i} \omega t} \tilde{\mu}_{\mathrm{qg}} \tilde{\varepsilon}_{q}(t) \varepsilon_{\mathrm{IR}}(t-\Delta t) \mathrm{e}^{\mathrm{i} I_{p} t} \mathrm{e}^{\mathrm{i} \Phi_{P}(t-\Delta t)} .
$$

This is a simplified version of second-order perturbation theory. The two photons are assumed to be absorbed at the same time and that only the lowest-order sidebands are included. The measurable quantity is $I_{\mathrm{SB}}(\omega, \Delta t)=\left|\mathcal{A}_{\mathrm{SB}}(\omega, \Delta t)\right|^{2}$, which is illustrated in figure 3 where ionization from the harmonic has also been included. Apart from the additional phase terms, both the equation and the resulting time-frequency representation strongly resembles the equation and traces used in frequency-resolved optical gating (FROG) measurements [31] with the IR probe acting as the gate function. This type of FROG measurement, where the pulse to be measured is cross-correlated with a reference pulse, will from here on be called XFROG in analogy with the usual FROG nomenclature. Just as FROG traces are used to 
characterize optical pulses, the sidebands can be used to characterize the time-frequency structure of the XUV pulse.

The sideband signal integrated over energy corresponds, in time, to the convolution of the temporal profiles of the XUV and IR pulses. Introducing, for Gaussian pulses, $r$ as the ratio $\tau_{\mathrm{IR}} / \tau_{q}$, where $\tau_{\mathrm{IR}}$ and $\tau_{q}$ are the durations of IR and XUV pulses respectively, the full width at half maximum of the sideband signal is given by [4]

$$
\tau_{\mathrm{SB}}=\tau_{q} \sqrt{1+r^{2}} .
$$

Therefore, by knowing the IR pulse duration the harmonic duration can be extracted from the energy integrated sideband. Reducing the probe duration improves the temporal resolution, but in the limit with the best temporal resolution, when $r \rightarrow 0$, no spectral resolution is possible. The choice of probe duration is, therefore, a compromise between the temporal and spectral resolution.

The position of the sideband in energy can vary as a function of time-delay between the two pulses for three reasons: (i) if there is a chirp on the XUV pulse, $b_{q}$; (ii) if there is a chirp on the IR probe; or (iii) if the IR intensity is sufficiently strong to introduce a ponderomotive shift. We are here mainly interested in the first reason, and the IR probe will therefore be assumed not to have a chirp and to be sufficiently weak that the ponderomotive shift can be neglected (we will later show the effects of an increased IR intensity). With these assumptions the tilt of the sideband is only due to the chirp of the XUV pulse and the resulting slope of the sideband (the variation of the peak energy with time) will be denoted $b_{\mathrm{SB}}$. Due to the duration of the probe, $b_{\mathrm{SB}} \neq b_{q}$. It can be seen from equation (6) that for a given time-delay, $\Delta t$, the sidebands are generated not mainly at the peak of the IR pulse but instead at $\Delta t /\left(1+r^{2}\right)$ where the product $F_{q}(t) F_{\mathrm{IR}}(t-\Delta t)$ is maximized. Knowing the probe duration, however, it is possible to calculate

$$
b_{q}=b_{\mathrm{SB}}\left[1+r^{2}\right] .
$$

In an experiment the IR pulse used as a probe has to be sufficiently strong to generate detectable sidebands and may therefore introduce a ponderomotive shift that has to be accounted for when the chirp rate is extracted [4]. For the sidebands, just as for the harmonic peak, the ponderomotive shift turns out to be determined not only by the IR intensity but also by the relative duration between the XUV and IR pulses. The sidebands are only generated when both pulses are present, however, and so the effect of the relative durations on the sideband position is different from the effect on the main peak. To analyse the effect of the IR intensity and duration on the generated sidebands, we calculate the average energy of the sideband

$$
\langle E\rangle=\frac{\int \mathrm{d} E E\left|\mathcal{A}_{\mathrm{SB}}(E)\right|^{2}}{\int \mathrm{d} E\left|\mathcal{A}_{\mathrm{SB}}(E)\right|^{2}} .
$$

For Gaussian pulses this can be written as the sum of two terms

$$
\langle E(\Delta t)\rangle=-U_{p} \sqrt{\frac{1+r^{2}}{2+r^{2}}} \mathrm{e}^{-4 \ln (2)\left(\Delta t / \tau_{d}\right)^{2}}+\frac{b_{q}}{1+r^{2}} \Delta t,
$$

where $U_{p}$ is the ponderomotive shift at the peak IR intensity and $\tau_{d}$ is a characteristic decay time given by

$$
\tau_{d}=\tau_{\mathrm{SB}} \sqrt{1+2 / r^{2}}
$$

There are several interesting things to notice here: (1) the harmonic chirp enters the equation in the second term, corrected for the relative duration, as described previously; (2) the ponderomotive shift is reduced with decreasing IR duration (the $\sqrt{\frac{1+r^{2}}{2+r^{2}}}$ factor) and (3) the 


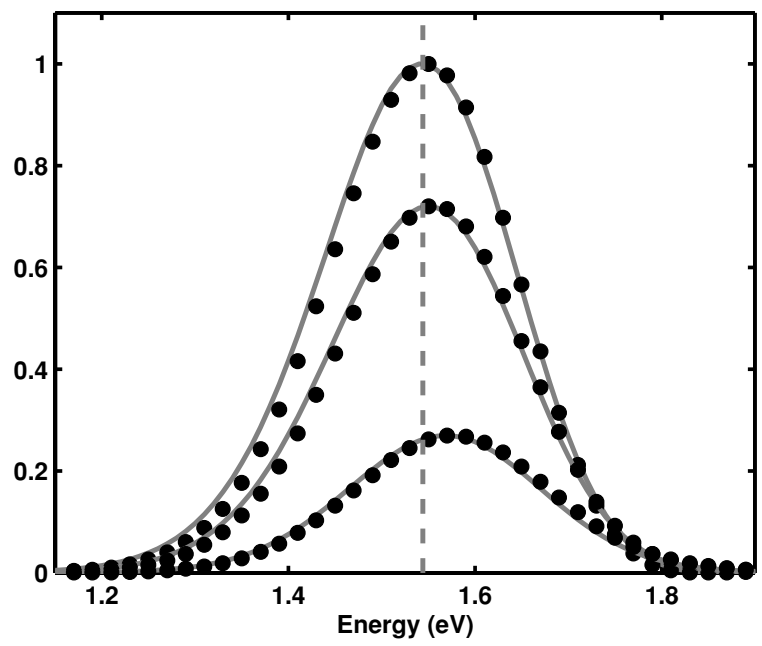

Figure 4. A comparison between the sideband spectra for three different time-delays obtained by solving the TDSE (symbols) and using the simplified model (lines). Both the IR pulse and the XUV pulse had a Gaussian pulse shape with a duration of 10.2 fs FWHM and the IR intensity was $10^{12} \mathrm{~W} \mathrm{~cm}^{-2}$.

characteristic decay time $\tau_{d}$ increases as $r$ decreases so that the sideband merely shifts while the shape remains more or less unaffected for small values of $r$ (experimental demonstration of this was recently published in [6]). This means that in the limit of a very short probe the chirp rate of the harmonic can be extracted directly from the experimental data. It should be noted that even though the ponderomotive shift is reduced, the effect on the sideband position is such that the separation between two sidebands remains $2 \omega_{1}$ and that the absorption and emission of an IR photon from two consecutive harmonics still yield the same final sideband energy. The advantages of using an IR probe that is shorter than the XUV pulse to be measured are:

(i) the duration of the XUV pulse can be determined more accurately (equation (7));

(ii) the chirp rate of the XUV pulse can be determined more accurately (equation (8));

(iii) the distortion of the sideband due to the ponderomotive shift is reduced (equation (10)).

The results presented above are based on perturbation theory. In order to confirm the validity of these results, we solve the time-dependent Schrödinger equation (TDSE) for the two-colour, multi-photon ionization. We do this by numerical integration within the single active electron approximation [34], using a pseudo-potential [35] to describe xenon. In figure 4 we compare the modelled sideband with the results from the TDSE calculation for three different time-delays. The agreement is very satisfactory, in particular the positions of the peaks agree very well (within a few per cent) when comparing the two calculations.

\section{Multi-colour, multi-photon photoelectron spectra}

In this section we expand the model described above, to take several harmonics into account simultaneously. We also demonstrate how the complete time structure of the harmonic emission can be reconstructed from measurements of the photoelectron spectra as a function of time-delay between the harmonics and the IR probe. The IR intensity used should not be too high, and a good rule of thumb is that the strengths of the sidebands should never exceed $50 \%$ of that of the principal peaks [39]. In this case each sideband is generated with contributions 


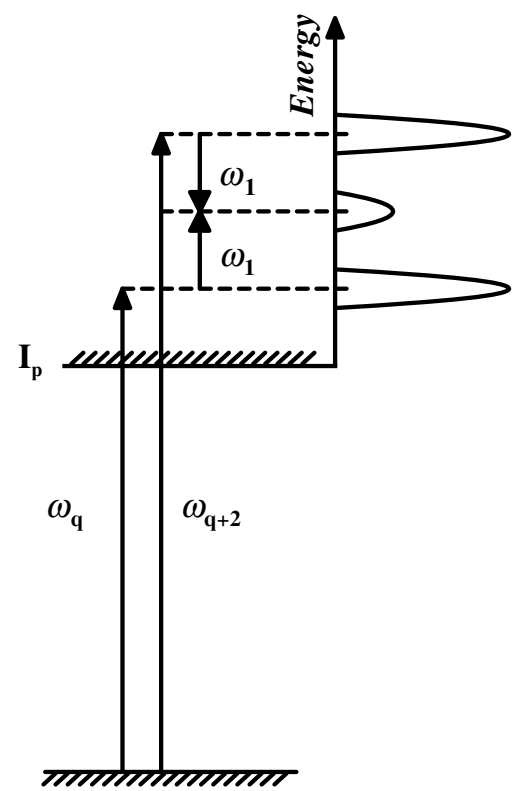

Figure 5. Schematic of three-colour, two-photon ionization. When two harmonics contribute to the ionization process, the intervening sideband is generated through two different ionization paths. These two paths interfere, which results in a modulation of the sideband intensity as a function of pump-probe delay.

from two consecutive harmonics (see figure 5), and higher-order sidebands can be neglected. Assuming that the IR intensity is kept sufficiently low, it is therefore enough to include only two harmonics in the model, since the total photoelectron spectrum can then be concatenated from these results.

The sideband spectrum containing contributions from two consecutive harmonics can be calculated using equation (6) if $\tilde{\mu}_{\mathrm{qg}} \tilde{\varepsilon}_{q}(t)$ is replaced by $\tilde{\mu}_{\mathrm{qg}} \tilde{\varepsilon}_{q}(t)+\tilde{\mu}_{\mathrm{q}+2 \mathrm{~g}} \tilde{\varepsilon}_{q+2}(t)$, i.e.

$$
\mathcal{A}_{\mathrm{SB}}\left(\delta \omega_{q}, \Delta t\right)=\int_{-\infty}^{\infty} \mathrm{d} t \mathrm{e}^{\mathrm{i} \delta \omega_{q} t}\left[\tilde{\mu}_{\mathrm{qg}} \tilde{\varepsilon}_{q}(t)+\tilde{\mu}_{\mathrm{q}+2 \mathrm{~g}} \tilde{\varepsilon}_{q+2}(t)\right] \varepsilon_{\mathrm{IR}}(t-\Delta t),
$$

where the ponderomotive shift has been left out and $\delta \omega_{q}=\omega-(q+1) \omega_{1}$, thereby centring the sideband around $\delta \omega_{q}=0$. Equation (12) can be used for arbitrarily shaped pulses, but we will still assume that the probe is transform limited. It is well known that the intensity of the sideband, which is given by $I_{\mathrm{SB}}=\left|\mathcal{A}_{\mathrm{SB}}\right|^{2}$, will be modulated as a function of pump-probe delay when the sideband is generated from two harmonics and that the relative phase between the harmonics can be extracted from this modulation [7, 24]. The modulation of the sideband intensity is the result of interference between the two ionization paths, and if the two harmonics that contribute to the sideband have no chirp (or, as we will argue, if they have the same chirp rate) the modulated part is given by

$$
I_{\mathrm{SB}}(\Delta t) \propto \cos \left(2 \omega_{1} \Delta t-\Delta \phi_{q+1}^{0}-\Delta \phi_{q+1}^{\mathrm{at}}\right),
$$

where $\Delta \phi_{q+1}^{0}=\phi_{q}^{0}-\phi_{q+2}^{0}$ is the relative phase between the contributing harmonics and $\Delta \phi_{q+1}^{\text {at }}=\phi_{q}^{\text {at }}-\phi_{q+2}^{\text {at }}$ is the difference in the characteristic atomic phases of the two ionization pathways [28]. Assuming that the atomic dipole phase can be calculated accurately $[38,39]$, the relative phase between the harmonics can be extracted from this modulation [7]. This description, however, assumes that the two harmonics have the same time-dependent 
frequency modulation, $\phi(t)$, which is usually not the case [6]. If consecutive harmonics have different frequency modulations, the timing between the pulses in the resulting APT will vary across the train [18]. In order to use an attosecond train in an experiment, knowledge of the pulse-to-pulse timing is crucial.

An example of photoelectron spectra at different time-delays, calculated using the strong field approximation [40], are shown on the left side of figure 6 for a realistic pulse train. Most of the sidebands show a pronounced tilt, i.e. the position of their peak energy varies with probe delay. From this tilt, the average chirp rate of the two contributing harmonics can be extracted, using the technique described in the previous section. To completely reconstruct the attosecond pulse train, however, we need the individual chirp rates, which requires that the difference in chirp rates between consecutive harmonics also can be measured.

Fortunately, the modulation of the sideband intensity is altered by the difference in chirp rates, which provides a means to measure this difference. This modulation varies as a function of both time (delay) and energy, but sufficient information is contained in one line-out per sideband, and from here on we will only consider the modulation at one energy per sideband, at $\delta \omega_{q}=0$. The total time-frequency representation of the sideband is derived in appendix for pulses with Gaussian envelopes. The physical origin of the change in sideband modulation can be understood as follows. When the chirp rates of the two harmonics are different, different IR frequencies are needed at different time-delays to reach the central energy of the sideband. With different IR frequencies used to generate the central part of the sideband, the periodicity of the modulation will vary with time-delay.

The change in the periodicity of the sideband modulation with probe delay can be accounted for if a time-dependent phase term, given by $-\beta \Delta b_{q+1} \frac{\Delta t^{2}}{2}$, is added to equation (13). $\Delta b_{q+1}$ is the sought for difference in chirp rates, and $\beta \neq 1$ is a result of the non-zero probe duration. $\beta$ approaches 1 when the probe duration approaches 0 . In the experimentally common limit where the bandwidth needed to account for the chirp rate is small compared to the bandwidth needed to account for the duration (i.e. $\left[b_{q} /(2 A)\right]^{2} \ll 1$, where $A=\frac{2 \ln 2}{\tau_{\mathrm{XUV}}^{2}}+\frac{2 \ln 2}{\tau_{\mathrm{IR}}^{2}}$ ) $\beta$ can be written as $\beta=\left[1 /\left(1+r^{2}\right)\right]^{2}$. This access to $\Delta b_{q+1}$ provides a sufficient means to retrieve the individual chirp rates of the two harmonics from their common sideband.

Unfortunately, an additional phase correction also needs to be added to equation (13) in order to accurately describe the effect of the difference in chirp rates, namely $-\Delta \phi_{q+1}^{\text {corr }}=$ $-\frac{1}{2}\left[\arctan \left(\frac{b_{q}}{2 A}\right)-\arctan \left(\frac{b_{q+2}}{2 A}\right)\right]$. This phase term introduces an overall phase shift of the sideband modulation and needs, just like the atomic dipole phase, to be accounted for if the time structure is to be reconstructed correctly. The magnitude of this correction decreases with decreasing probe duration. The final expression for the modulated part of the sideband signal at $\delta \omega_{q}=0$ reads

$$
I_{\mathrm{SB}}(\Delta t) \propto \cos \left(2 \omega_{1} \Delta t-\Delta \phi_{q+1}^{0}-\Delta \phi_{q+1}^{\mathrm{at}}-\Delta \phi_{q+1}^{\mathrm{corr}}-\beta \Delta b_{q+1} \frac{\Delta t^{2}}{2}\right) .
$$

This equation has a structure similar to the SPIDER equation ([32]) and the same algorithm that is used to retrieve the spectral phase in a SPIDER measurement can be used to extract $\Delta b_{q+1}$, thereby making the phase retrieval very fast and robust. The time-independent phase difference, $\Delta \phi_{q+1}^{0}$, can still be retrieved using the same procedure as is used when the harmonics are assumed to have the same chirp rates.

An example of a reconstruction using TIGERS is shown in figure 6 for a realistic pulse train. The chirp rates of harmonics 13 through 23 generated in argon by a $20 \mathrm{fs}, 800 \mathrm{~nm}$ IR pulse with an intensity of $1.5 \times 10^{14} \mathrm{~W} \mathrm{~cm}^{-2}$ are calculated using the strong field approximation. The strong field approximation is also used to calculate the photoelectron spectra at different time-delays when these harmonics are used to ionize argon in the presence of an IR field. 

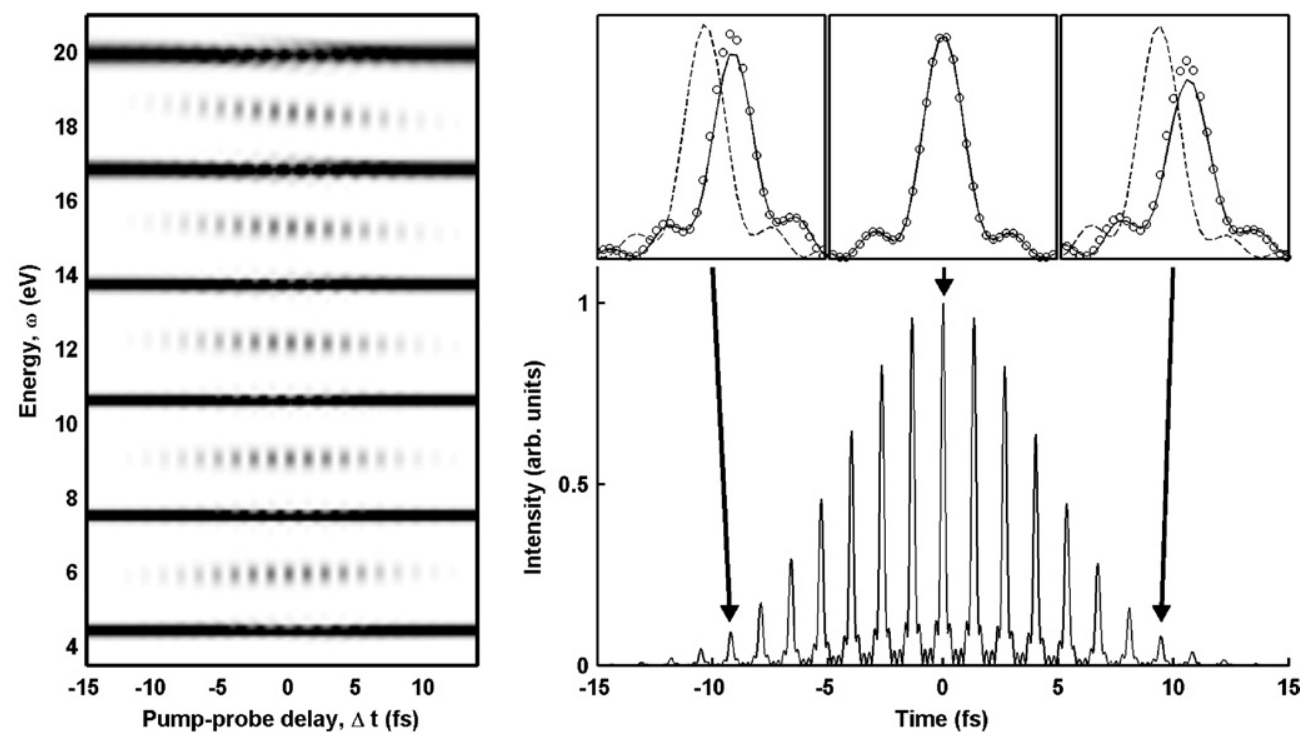

Figure 6. Reconstruction of an attosecond pulse train. To the left the calculated delay-dependent photoelectron spectrum from argon is shown for harmonics 13 to 23 and an IR probe of 5 fs duration. The sidebands exhibit a tilt like in figure 3 , here increasing with order, but also a modulation due to the interference between pathways. To the right the pulse train reconstructed using the method described in the text is shown. The insets show magnified parts of the time structure in the beginning, the centre, and the end of the train respectively. In the insets the solid line shows the true time structure, the circles show the result of the reconstruction and the dashed line shows the result of a reconstruction where the harmonic chirp has been ignored.

The duration of the IR probe is $5 \mathrm{fs}$ and the harmonic durations are $10 \mathrm{fs}$, which gives $r=\frac{1}{2}$ and $\left(b_{q} / 2 A\right)^{2}$ does not exceed 0.16 for any of the harmonics. From the tilt in energy of the sidebands as a function of pump-probe delay, the averaged chirp rates of the harmonics are extracted. They get increasingly more negative with increasing harmonic order. $\Delta \phi_{q+1}$ and $\Delta b_{q+1}$ are calculated from line-outs at $\delta \omega_{q}=0$ for each sideband. The change in the chirp rate from one harmonic to the next leads in the time-domain to a variation of the periodicity of the pulses in the train [6]. This effect is overlooked if the harmonic chirp rates are ignored in the reconstruction (dashed line on top of figure 6), but accurately accounted for using TIGERS (solid line and circles on top of figure 6).

In most cases the phase correction factors in equation (14) are rather small, even though probe pulses longer than the individual harmonic pulses are frequently used, since the chirp rates of consecutive harmonics usually vary only slowly with the harmonic order. It is therefore a good approximation to use equation (13) when measuring the duration of the pulses in an APT. However, if these techniques are to be used, for instance, to measure the small contributions from the atomic phases [39], the additional corrections have to be known and accounted for. Also, if one wants to tailor the attosecond pulse trains using, for instance, chirped XUV optics, the corrections may very well be important.

\section{Conclusions}

In this paper we have summarized the multi-photon, multi-colour photoelectron spectroscopy techniques used to characterize the time-frequency structure of ultrashort XUV pulses produced by high-order harmonic generation. We have demonstrated the advantages of using 
a probe pulse that is shorter than the XUV pulse to be measured (or shorter than the individual harmonics that produce the APT). An analytic expression has been derived for the total timefrequency representation of a sideband generated from two consecutive harmonics. Based on this expression, it has been shown that it is possible to accurately characterize individual harmonics even when the sideband used originates from two different harmonics, and that it is possible to completely reconstruct the entire temporal structure of the APT.

\section{Acknowledgments}

We acknowledge support from the National Science Foundation through grant no PHY0401625. We also acknowledge the support of the Integrated Initiative of Infrastructure Laserlab-Europe (RII3-CT-2003-506350, FOSCIL), the Marie Curie Intra-European Fellowship (MEIF-CT-2004-009268), the Marie Curie Research Training Network XTRA (MRTN-CT-2003-505138) and the Swedish Science Council. KV is on leave from the Department of Optics and Quantum Electronics, University of Szeged, Hungary and RL-M is currently at Laboratoire d'Optique Appliquée, ENSTA, France.

\section{Appendix. Derivation and definitions}

The time-frequency representation of a sideband generated with contributions from two harmonics can be calculated numerically for arbitrary pulse shapes, but the underlying physics is easier to understand if we can derive an analytic expression. Assuming that the contributing harmonics and the IR probe have Gaussian envelopes, it is possible to derive such an expression. We furthermore assume that the IR probe has a transform limited pulse duration and that the frequency modulation of the harmonics can be described as linear chirps. With these assumptions the four fields used in the derivation can be expressed as

$$
\left\{\begin{array}{l}
\tilde{\varepsilon}_{q}(t)=\exp \left(-a_{\mathrm{XUV}} t^{2}\right) \exp \left(-\mathrm{i} q \omega_{1} t-\mathrm{i} \phi_{q}^{0}-\mathrm{i} b_{q} \frac{t^{2}}{2}\right) \\
\tilde{\varepsilon}_{q+2}(t)=\exp \left(-a_{\mathrm{XUV}} t^{2}\right) \exp \left(-\mathrm{i}(q+2) \omega_{1} t-\mathrm{i} \phi_{q+2}^{0}-\mathrm{i} b_{q+2} \frac{t^{2}}{2}\right) \\
\tilde{\varepsilon}_{\mathrm{IR}}^{+}(t)=\exp \left(-a_{\mathrm{IR}} t^{2}\right) \exp \left(-\mathrm{i} \omega_{1} t\right) \\
\tilde{\varepsilon}_{\mathrm{IR}}^{-}(t)=\exp \left(-a_{\mathrm{IR}} t^{2}\right) \exp \left(+\mathrm{i} \omega_{1} t\right)
\end{array}\right.
$$

where $a_{\mathrm{XUV}}=\frac{2 \ln 2}{\tau_{\mathrm{XUV}}^{2}}, a_{\mathrm{IR}}=\frac{2 \ln 2}{\tau_{\mathrm{IR}}^{2}}$. The IR field, $\varepsilon_{\mathrm{IR}}=\tilde{\varepsilon}_{\mathrm{IR}}^{+}+\tilde{\varepsilon}_{\mathrm{IR}}^{-}$, is real, but the use of complex notation distinguishes between the absorption of an additional IR photon, $\tilde{\varepsilon}_{\mathrm{IR}}^{+}$, and the stimulated emission of one, $\tilde{\varepsilon}_{\mathrm{IR}}^{-}$.

The following definitions are used in the derivation:

$$
\left\{\begin{array}{l}
A=a_{\mathrm{XUV}}+a_{\mathrm{IR}} \\
K=\sqrt{\pi} \mathrm{e}^{-a_{\mathrm{IR}} \Delta t^{2}} \\
\phi_{j}^{\mathrm{corr}}=\frac{1}{2} \arctan \left(\frac{b_{j}}{2 A}\right) \\
W_{j}^{-1}=\sqrt[4]{A^{2}+\frac{b_{j}^{2}}{4}} \\
M_{j}(\omega, \Delta t)=\left|\mu_{j g}\right| W_{j} \exp \left(W_{j}^{4}\left(\frac{a_{\mathrm{IR}} \Delta t \omega b_{j}}{2}-\frac{\omega^{2} A}{4}+A a_{\mathrm{IR}}^{2} \Delta t^{2}\right)\right) \\
Q_{j}(\omega)=W_{j}^{4} \omega^{2} b_{j} / 8 \\
S_{j}(\omega, \Delta t)=W_{j}^{4} a_{\mathrm{IR}} A \omega \Delta t
\end{array}\right.
$$

where the indices $j$ label the harmonics. 
We are interested in finding an expression for the $(q+1)$ th sideband and will assume that the IR intensity is so low that it is sufficient to include only the two harmonics with frequencies $q \omega_{1}$ and $(q+2) \omega_{1}$, from here on labelled 0 and 2 respectively. Using perturbation theory, the amplitude of the sideband can be expressed as

$$
\mathcal{A}_{\mathrm{SB}}(\omega, \Delta t)=\int_{-\infty}^{\infty} \mathrm{d} t \mathrm{e}^{\mathrm{i} \omega t}\left[\tilde{\mu}_{0 \mathrm{~g}} \tilde{\varepsilon}_{0}(t)+\tilde{\mu}_{2 \mathrm{~g}} \tilde{\varepsilon}_{2}(t)\right] \varepsilon_{\mathrm{IR}}(t-\Delta t),
$$

where the ponderomotive shift and the ionization potential have been left out for simplicity. By replacing $\omega$ with the frequency difference $\delta \omega_{q}=\omega-(q+1) \omega_{1}$ and using the definitions for the fields (A.1), equation (A.3) can be written as

$$
\begin{aligned}
\mathcal{A}_{\mathrm{SB}}\left(\delta \omega_{q}, \Delta t\right)= & \int_{-\infty}^{\infty} \mathrm{d} t \mathrm{e}^{\mathrm{i} \delta \omega_{q} t} \mathrm{e}^{\mathrm{i}(q+1) \omega_{1} t}\left\{\tilde{\mu}_{0 \mathrm{~g}} \tilde{\varepsilon}_{0}(t) \tilde{\varepsilon}_{\mathrm{IR}}^{+}(t-\Delta t)+\tilde{\mu}_{2 \mathrm{~g}} \tilde{\varepsilon}_{2}(t) \tilde{\varepsilon}_{\mathrm{IR}}^{-}(t-\Delta t)\right\} \\
= & \mathrm{e}^{-a_{\mathrm{IR}} \Delta t^{2}} \int_{-\infty}^{\infty} \mathrm{d} t \mathrm{e}^{\mathrm{i} \delta \omega_{q} t} \mathrm{e}^{2 a_{\mathrm{IR}} \Delta t \cdot t-A t^{2}}\left\{\tilde{\mu}_{0 \mathrm{~g}} \exp \left(-\mathrm{i}\left[\phi_{0}^{0}+b_{0} \frac{t^{2}}{2}-\omega_{1} \Delta t\right]\right)\right. \\
& \left.+\tilde{\mu}_{2 \mathrm{~g}} \exp \left(-\mathrm{i}\left[\phi_{2}^{0}+b_{2} \frac{t^{2}}{2}+\omega_{1} \Delta t\right]\right)\right\} \\
= & \mathrm{e}^{-a_{\mathrm{IR}} \Delta t^{2}} \int_{-\infty}^{\infty} \mathrm{d} t\left\{\tilde { \mu } _ { 0 \mathrm { g } } \operatorname { e x p } ( - \mathrm { i } [ \phi _ { 0 } ^ { 0 } - \omega _ { 1 } \Delta t ] ) \cdot \operatorname { e x p } \left(-\left[A+\mathrm{i} \frac{b_{0}}{2}\right] t^{2}\right.\right. \\
& \left.+\mathrm{i}\left[\delta \omega_{q}-2 \mathrm{i} a_{\mathrm{IR}} \Delta t\right] t\right)+\tilde{\mu}_{2 \mathrm{~g}} \exp \left(-\mathrm{i}\left[\phi_{2}^{0}+\omega_{1} \Delta t\right]\right) \\
& \left.\times \exp \left(-\left[A+\mathrm{i} \frac{b_{2}}{2}\right] t^{2}+\mathrm{i}\left[\delta \omega_{q}-2 \mathrm{i} a_{\mathrm{IR}} \Delta t\right] t\right)\right\}
\end{aligned}
$$

Integration leads to

$$
\begin{aligned}
\mathcal{A}_{\mathrm{SB}}\left(\delta \omega_{q}, \Delta t\right)= & \mathrm{e}^{-a_{\mathrm{IR}} \Delta t^{2}}\left\{\tilde{\mu}_{0 \mathrm{~g}} \exp \left(-\mathrm{i}\left[\phi_{0}^{0}-\omega_{1} \Delta t\right]\right) \cdot \sqrt{\frac{\pi}{A+\mathrm{i} \frac{b_{0}}{2}}}\right. \\
& \times \exp \left(-\frac{\left(\delta \omega_{q}-2 \mathrm{i} a_{\mathrm{IR}} \Delta t\right)^{2}}{4\left(A+\mathrm{i} \frac{b_{0}}{2}\right)}\right)+\tilde{\mu}_{2 \mathrm{~g}} \exp \left(-\mathrm{i}\left[\phi_{2}^{0}+\omega_{1} \Delta t\right]\right) \\
& \left.\times \sqrt{\frac{\pi}{A+\mathrm{i} \frac{b_{2}}{2}}} \cdot \exp \left(-\frac{\left(\delta \omega_{q}-2 \mathrm{i} a_{\mathrm{IR}} \Delta t\right)^{2}}{4\left(A+\mathrm{i} \frac{b_{2}}{2}\right)}\right)\right\},
\end{aligned}
$$

which can be further simplified by using

$$
\sqrt{\frac{1}{A+\mathrm{i} b_{j} / 2}}=W_{j} \times \mathrm{e}^{-\mathrm{i} \phi_{j}^{\text {corr }}}
$$

and some more definitions from (A.2) to give

$$
\begin{aligned}
\mathcal{A}_{\mathrm{SB}}\left(\delta \omega_{q}, \Delta t\right) & =K\left\{\mu_{0 g} W_{0} \exp \left(-\mathrm{i} \phi_{0}^{\text {corr }}\right) \exp \left(-\frac{W_{0}^{4}}{4}\left(A-\mathrm{i} b_{0} / 2\right)\left(\delta \omega_{q}-2 \mathrm{i} a \Delta t\right)^{2}\right)\right. \\
& \times \exp \left(\mathrm{i}\left(\omega_{1} \Delta t-\phi_{0}\right)\right)+\mu_{2 g} W_{2} \exp \left(-\mathrm{i} \phi_{2}^{\text {corr }}\right) \\
& \left.\times \exp \left(-\frac{W_{2}^{4}}{4}\left(A-\mathrm{i} b_{2} / 2\right)\left(\delta \omega_{q}-2 \mathrm{i} a \Delta t\right)^{2}\right) \exp \left(-\mathrm{i}\left(\omega_{1} \Delta t+\phi_{2}\right)\right)\right\} .
\end{aligned}
$$


With some algebra and a few more definitions from (A.2), this expression can be further rewritten as

$$
\begin{aligned}
\mathcal{A}_{\mathrm{SB}}\left(\delta \omega_{q}, \Delta t\right)= & K\left\{M_{0} \exp \left(-\mathrm{i} \phi_{0}^{\text {at }}\right) \exp \left(-\mathrm{i} \phi_{0}^{\text {corr }}\right) \exp \left(\mathrm{i}\left(\omega_{1} \Delta t-\phi_{0}-W_{0}^{4} a^{2} b_{0} \Delta t^{2} / 2\right)\right)\right. \\
& \times \exp \left(\mathrm{i}\left(Q_{0}+S_{0}\right)\right)+M_{2} \exp \left(-\phi_{2}^{\text {at }}\right) \exp \left(-\mathrm{i} \phi_{2}^{\text {corr }}\right) \\
& \left.\times \exp \left(\mathrm{i}\left(-\omega_{1} \Delta t-\phi_{2}-W_{2}^{4} a^{2} b_{0} \Delta t^{2} / 2\right)\right) \exp \left(\mathrm{i}\left(Q_{2}+S_{2}\right)\right)\right\}
\end{aligned}
$$

From this expression the intensity of the sideband, which is given by

$$
I_{\mathrm{SB}}\left(\delta \omega_{q}, \Delta t\right)=\mathcal{A}_{\mathrm{SB}} \times \mathcal{A}_{\mathrm{SB}}^{*},
$$

can be calculated. The final expression for the time-frequency representation of the sideband is

$$
\begin{aligned}
I_{\mathrm{SB}}\left(\delta \omega_{q}, \Delta t\right)= & K^{2}\left\{M_{0}^{2}+M_{2}^{2}+2 M_{0} M_{2} \cos \left(2 \omega_{1} \Delta t-\Delta \phi_{1}-\Delta \phi_{1}^{\mathrm{at}}-\Delta \phi_{1}^{\mathrm{corr}}\right.\right. \\
& \left.\left.-\frac{a^{2} \Delta t^{2}}{2}\left[W_{0}^{4} b_{0}-W_{2}^{4} b_{2}\right]+\Delta Q_{1}+\Delta S_{1}\right)\right\},
\end{aligned}
$$

where all differences are defined as

$$
\Delta X_{1}=X_{0}-X_{2} \text {. }
$$

Many of the terms in equation (A.12) are needed to describe the total time-energy structure of the sideband, and the equation is significantly simplified if it is only evaluated for $\delta \omega_{q}=0$, in which case the modulation of the sideband intensity is given by

$$
I_{\mathrm{SB}}\left(\delta \omega_{q}, \Delta t\right) \propto \cos \left(2 \omega_{1} \Delta t-\Delta \phi_{1}-\Delta \phi_{1}^{\mathrm{at}}-\Delta \phi_{1}^{\mathrm{corr}}-\frac{a^{2} \Delta t^{2}}{2}\left[W_{0}^{4} b_{0}-W_{2}^{4} b_{2}\right]\right) .
$$

This equation still includes sufficient information to retrieve $\Delta b_{1}$ since the different chirp rates are included in the derivation.

\section{References}

[1] Ferray M, L’Huillier A, Li X F, Lompré L A, Mainfray G and Manus C 1988 J. Phys. B: At. Mol. Opt. Phys. 21 L31

[2] Agostini P and DiMauro L 2004 Rep. Prog. Phys. 67813

[3] Krause J L, Schafer K J and Kulander K C 1992 Phys. Rev. Lett. 683535

[4] Norin J et al 2002 Phys. Rev. Lett. 88193901

[5] Sekikawa T, Katsura T, Miura S and Watanabe S 2002 Phys. Rev. Lett. 88193902

[6] Mauritsson J, Johnsson P, López-Martens R, Varjú K, Kornelis W, Biegert J, Keller U, Gaarde M B, Schafer K J and L'Huillier A 2004 Phys. Rev. A 70 021801(R)

[7] Paul P M, Toma E S, Breger P, Mullot G, Augé F, Balcou Ph, Muller H G and Agostini P 2001 Science 292 1689

[8] Mairesse Y et al 2003 Science 3021540

[9] Tzallas P, Charalambidis D, Papadogiannis N A, Witte K and Tsakiris G D 2003 Nature 426267

[10] Aseyev S A, Ni Y, Frasinski L J, Muller H G and Vrakking M J J 2003 Phys. Rev. Lett. 91223902

[11] López-Martens R et al 2005 Phys. Rev. Lett. 94033001

[12] López-Martens R, Mauritsson J, Johnsson P, Varjú K, L’Huillier A, Kornelis W, Biegert J, Keller U, Gaarde M and Schafer K 2004 Appl. Phys. B 78835

[13] Hentschel M, Kienberger R, Spielmann Ch, Reider G A, Milosevic N, Brabec T, Corkum P, Heinzmann U, Drescher M and Krausz F 2001 Nature $\mathbf{4 1 4} 509$

[14] Drescher M, Hentschel M, Kienberger R, Uiberacker M, Yakovlev V, Scrinzi A, Westerwalbesloh Th, Kleineberg U, Heinzmann U and Krausz F 2002 Nature 419803

[15] Nugent-Glandorf L, Scheer M, Samuels D A, Mulhisen A M, Grant E R, Yang X, Bierbaum V M and Leone S R 2001 Phys. Rev. Lett. 87193002

[16] Zamith S et al 2003 J. Chem. Phys. 1193763 
[17] Johnsson P et al 2005 Phys. Rev. Lett. at press

[18] Varjú K et al 2005 J. Mod. Opt. 52379

[19] Gaarde M 2001 Opt. Exp. 8529

[20] Schafer K J, Yang B, DiMauro L F and Kulander K C 1993 Phys. Rev. Lett. 701599

[21] Corkum P B 1993 Phys. Rev. Lett. 711994

[22] Lewenstein M, Salières P and L'Huillier A 1995 Phys. Rev. A 524747

[23] Drescher M, Hentschel M, Kienberger R, Tempea G, Spielmann C, Reider G A, Corkum P B and Krausz F 2001 Science 2911923

[24] Muller H G 2002 Appl. Phys. B 74 S17

[25] Véniard V, Taïeb R and Maquet A 1995 Phys. Rev. Lett. 744161

[26] Glover T E, Schoenlein R W, Chin A H and Shank C V 1996 Phys. Rev. Lett. 762468

[27] Schins J M, Breger P, Agostini P, Constantinescu R C, Muller H G, Bouhal A, Grillon G, Antonetti A and Mysyrowicz A 1997 J. Opt. Soc. Am. B 13197

[28] Véniard V, Taïeb R and Maquet A 1996 Phys. Rev. A 54721

[29] Bucksbaum P H, Freeman R R, Bashkansky M and McIlratht T J 1987 J. Opt. Soc. Am. B 4760

[30] Freeman R R and Bucksbaum P H 1991 J. Phys. B: At. Mol. Opt. Phys. 24325

[31] Trebino R, DeLong K W, Fittinghoff D N, Sweetser J N, Krumbügel M A, Richman B A and Kane D J 1997 Rev. Sci. Instrum. $\mathbf{6 8} 1$

[32] Iaconis C and Walmsley I A 1998 Opt. Lett. 23792

[33] Toma E S, Muller H G, Paul P M, Breger P, Cheret M, Agostini P, Le Blanc C, Mullot G and Cheriaux G 2000 Phys. Rev. A 62 061801(R)

[34] Schafer K J and Kulander K C 1997 Phys. Rev. Lett. 78638

[35] Kulander K C and Rescigno T N 1991 Comput. Phys. Commun. 63523

[36] Reiss H R 1980 Phys. Rev. A 221786

[37] Schafer K J, Mauritsson J, Johnsson P and L'Huillier A in preparation

[38] Toma E S and Muller H G 2002 J. Phys. B: At. Mol. Opt. Phys. 353435

[39] Mauritsson J, Gaarde M B and Schafer K J 2005 Phys. Rev. A at press

[40] Lewenstein M, Balcou Ph, Ivanov M, L'Huillier A and Corkum P B 1994 Phys. Rev. A 492117 